\title{
Investigation of nonlinear 2D bottom transportation dynamics in coastal zone on optimal curvilinear boundary adaptive grids
}

\author{
Alexander Sukhinov ${ }^{1, *}$, Alexander Chistyakov ${ }^{1}$, and Valentina Sidoryakina ${ }^{2}$ \\ ${ }^{1}$ Don State Technical University, Rostov-on-Don, Russian Federation \\ ${ }^{2}$ Taganrog Institute after A.P. Chekhov (branch) RGEU (RINE), Taganrog, Russian Federation
}

\begin{abstract}
One of the practically important tasks of hydrophysics for sea coastal systems is the problem of modeling and forecasting bottom sediment transportation. A number of problems connected to ship safety traffic, water medium condition near the coastal line etc. depends on forecasting bottom deposit transportation under natural and technogenic influences. Coastal systems are characterized by a complicated form of coastline - the presence of long, narrow and curvilinear peninsulas and bays. Water currents and waves near the beach are strongly depend on complicated coastal line and in turn, exert on the bottom sediment transportation near the shore. The use of rectangular grids in the construction of discrete models leads to significant errors in both the specification of boundary conditions and in the modeling of hydrophysical processes in the coastal zone. In this paper, we consider the construction of a finite-element approximation of the initial-boundary value problem for the spatially two-dimensional linearized equation of sediment transportation using optimal boundary-adaptive grid. First, the linearization of a spatially twodimensional nonlinear parabolic equation on the time grid is performed-when the coefficients of the equation that are nonlinearly dependent on the bottom relief function are set on the previous time layer, and the corresponding initial conditions are used on the first time layer. The algorithm for constructing the grid is based on the procedure for minimizing the generalized Dirichlet functional. On the constructed grid, finite element approximation using bilinear basis functions is performed, which completes the construction of a discrete model for the given problem. The using of curvilinear boundary adaptive grids leads to decreasing of total grid number in 5-20 times and respectively the total modeling time and/or it allows to improve modeling accuracy.
\end{abstract}

\section{Introduction}

One of the essential features of coastal and marine systems is complicated form of the coastline, which hardly influences on mathematical modelling of these systems. The use of rectangular, does not allow to take into account, with practical value accuracy, the presence narrow sea coastal bodies of complicated shape, such as bays, estuaries, straits, peninsulas etc. At the same time, the presence of these objects hardly determines the structure of coastal currents, especially for shallow water bodies of the South of Russia, similar to the Azov Sea and Taganrog bay. The development of optimal curvilinear grids, consistent with the complex shape of the shoreline (boundary-adaptive grids), as shown by the practice of numerical solution of problems of this class, can significantly improve the accuracy of the solution obtained and / or reduce the amount of computation. The paper deals with the construction of a finite-element approximation of the initial-boundary value problem for the spatially two-dimensional linearized equation of sediment transport on a boundary-adaptive grid. First, the linearization of a spatially two-dimensional nonlinear parabolic equation on the time grid is performed, when the coefficients of the equation that depend nonlinearly on the bottom depth function are set on the previous time layer. The rationale for this method of linearization (the correctness of the formulation of the problem) and the convergence of the solutions of the linearized chain of problems in the norm of the space $\mathrm{L}^{1}$ with the step of the time grid tending to 0 was justified in the previous works of the authors [1-8]. The algorithm for constructing the grid is based on the procedure for minimizing the generalized Dirichlet functional. Previously, this approach has shown its effectiveness in the construction of minimal non degenerate grids for Z-shaped test regions, such as the "Maltese cross", etc., as well as in the numerical solution of problems of hydrodynamics of coastal systems, including in the Azov Sea [9]. On the constructed grid, in this paper, the finite element method is approximated using the bilinear basis functions of the linearized chain of sediment transport problems applied to the Azov Sea and the Taganrog Bay.

\footnotetext{
*Corresponding author: sukhinov@gmail.com
} 


\section{The formulation of the nonlinear initial-boundary value problem for two- dimensional models of sediment transport}

Let us consider the equation of sediment transport according to the [10-15]:

$$
(1-\varepsilon) \frac{\partial H}{\partial t}=\operatorname{div}\left(k \frac{\tau_{b c}}{\sin \varphi_{0}} \operatorname{grad} H\right)-\operatorname{div}\left(k \boldsymbol{\tau}_{b}\right),
$$

where $H=H(x, y, t)$ is the water depth; $\varepsilon$ is the porosity of bottom materials; $\boldsymbol{\tau}_{b}$ is the vector of tangential stress at the water bottom; $\tau_{b c}$ is a critical value of the tangential stress; $\tau_{b c}=a \sin \varphi_{0}, \varphi_{0}$ is an angle of repose of soil in the water; $k=k(H, x, y, t)$ is the nonlinear coefficient, determined by the ratio:

$$
k \equiv \frac{A \tilde{\omega} d}{\left(\left(\rho_{1}-\rho_{0}\right) g d\right)^{\beta}}\left|\boldsymbol{\tau}_{b}-\frac{\tau_{b c}}{\sin \varphi_{0}} \operatorname{gradH} H\right|^{\beta-1},
$$

where $\rho_{1}, \rho_{0}$ are the density of bottom material and water particles respectively; $g$ is the gravity acceleration; $\tilde{\omega}$ is the wave frequency; $A$ and $\beta$ are the dimensionless constants; $d$ are characteristic dimensions of soil particles.

Let's $D \subset R^{n}$ is an area where a process occurs, and $S$ is boundary with a piecewise smooth line. We considered a cylinder $L_{T}=D \times(0, T)$ of height $T$ with the base $D$ as the definitional domain of the equation (1). Its border consists of the lateral surface $S \times[0, T]$ and two bases: the upper base $\bar{D} \times\{0\}$ and the lower base $\bar{D} \times\{T\}$. Further, for simplicity, the equation (1) was considered in a rectangular domain $D(x, y)=\left\{0<x<L_{x}, 0<y<L_{y}\right\}$.

We added the initial condition to the equation (1), assuming that the initial conditions of function belong to the corresponding class of smoothness:

$$
\begin{gathered}
H(x, y, 0)=H_{0}(x, y), H_{0}(x, y) \in C^{2}(D) \cap C(\bar{D}), \\
\operatorname{grad}_{(x, y)} H_{0} \in C(\bar{D}),(x, y) \in \bar{D} .
\end{gathered}
$$

The conditions on the border domain $\bar{D}$ is following:

$$
\begin{gathered}
\mid \boldsymbol{\tau}_{b} \|_{y=0}=0, \\
H\left(L_{x}, y, t\right)=H_{2}(y, t), 0 \leq y \leq L_{y}, \\
H(0, y, t)=H_{1}(y, t), 0 \leq y \leq L_{y}, \\
H(x, 0, t)=H_{3}(x), 0 \leq x \leq L_{x},
\end{gathered}
$$

$$
H\left(x, L_{y}^{\prime}, t\right)=H_{4}(x, t), 0 \leq x \leq L_{x}, L_{y}^{\prime}<L_{y} .
$$

In addition to the boundary conditions (5)-(8), we assume the fulfillment of their smoothness conditions the existence of continuous derivatives on the boundary of the domain $\bar{D}$ :

$$
\operatorname{grad}_{(x, y)} H \in C\left(\bar{L}_{T}\right) \cap C^{1}\left(L_{T}\right) .
$$

We consider that there's a liquid layer of finite thickness always in this area and occur for the specified time interval at no drainage area, i.e.

$$
H(x, y, t) \geq c_{0} \equiv \text { const }>0,0 \leq x \leq L_{x}, 0 \leq y \leq L_{y}^{\prime}, 0 \leq t \leq T .
$$

The condition of non-degeneracy of the operator has the form:

$$
k \geq k_{0}=\text { const }>0, \forall(x, y) \in \bar{D}, 0<t \leq T .
$$

The vector of tangential stress at the bottom is calculated using the unit vectors of the coordinate system:

$$
\boldsymbol{\tau}_{b}=\bar{i} \tau_{b x}+\bar{j}_{b y}, \tau_{b x}=\tau_{b x}(x, y, t), \tau_{b y}=\tau_{b y}(x, y, t) .
$$

\section{Linearization of the sediment transportation equation on the time grid}

We will assume that the time interval $[0, T]$ is divided into time layers by values $t_{n}, n=1, \ldots, N$.

The values of the required function $H(x, y, t)$ and coefficient $k$ of the differential equation (1) on the $n$-th time layer (i.e. at $t=t_{n}$ ) will be denoted by $H^{n}(x, y, t)$ and $k^{n}$ respectively. These quantities are no longer timedependent $t$, but remain functions of spatial coordinates, i.e.

$$
H^{n}=H^{n}(x, y)=H^{n}\left(x, y, t_{n}\right), k^{n}=k^{n}(x, y)=k^{n}\left(x, y, t_{n}\right) .
$$

Further consideration of the problem is carried out on two time layers: the lower one $t=t_{n-1}$, on which the distribution of the unknown function $H(x, y, t)$ is known, (when $n=0$ the distribution is determined by the initial condition (3) $H^{0}(x, y)$ ), and the upper temporal layer $t=t_{n}$, on which the distribution of the desired function $H^{n}(x, y)$.

Reasoning is similarly repeated for any layer $t_{n}, n=1, \ldots, N$.

When $t=t_{n}$ equation (1) is written in the form:

$$
(1-\varepsilon) \frac{\partial H^{n}}{\partial t}-\operatorname{div}\left(k^{n-1} \frac{\tau_{b c}}{\sin \varphi_{0}} \operatorname{gradH} H^{n}\right)=-\operatorname{div}\left(k^{n-1} \boldsymbol{\tau}_{b}\right),
$$


and we supplement it with the initial conditions:

$$
\begin{gathered}
H^{1}\left(x, y, t_{0}\right)=H_{0}(x, y), H^{n}\left(x, y, t_{n-1}\right)=H^{n-1}\left(x, y, t_{n-1}\right), \\
(x, y) \in \bar{D}, n=2, \ldots, N .
\end{gathered}
$$

Member of the species $\operatorname{div}\left(k^{n-1} \boldsymbol{\tau}_{b}\right)$ will be a known function of the right-hand side. The boundary conditions (3) - (11) are assumed to be satisfied for any $t=t_{n}$, $n=1, \ldots, N$.

We also assume that the functions $H_{0}(x, y)$ and $H^{1}\left(x, y, t_{0}\right)$ from the initial conditions (14) are given by their values at the nodes of the spatial grid.

Consider the approximation of the differential equation (16) in time using the implicit scheme:

$$
\begin{gathered}
(1-\varepsilon) \frac{H^{n}-H^{n-1}}{\tau}=\operatorname{div}\left(k^{n-1} \frac{\tau_{b c}}{\sin \varphi_{0}} \operatorname{grad} H^{n}\right)-\operatorname{div}\left(k^{n-1} \boldsymbol{\tau}_{b}\right), \\
n=1, \ldots, N
\end{gathered}
$$

where $\tau=t^{n}-t^{n-1}$.

When the time is sampled for the parameter $\tau$ a limitation is imposed on the type of the Courant criterion.

\section{The construction of a discrete bottom sediment transportation model using the finite element method}

\subsection{The method of constructing an optimal boundary-adaptive grid (based on the example of the Sea of Azov and Taganrog Bay)}

Then the optimal boundary-adaptive grid, constructed for the water area of the Azov Sea and Taganrog Bay, is used in the calculations. The grid is considered as a necessary auxiliary step in solving the problem of sediment transport using the finite element method.

To construct the grid, we used cartographic differential operators and the corresponding net functionals described in [16-18].

In the plane $(x, y)$ a grid is under construction $\omega=\left\{\left(x_{i, j}, y_{i, j}\right), i=\overline{1, N_{1}}, j=\overline{1, N_{2}}\right\}$ by the given coordinates of the boundary nodes. The grid area separates $D$ on elementary subdomains in the form of quadrangles. Then we move from the variables $x, y$ to variables $\xi, \eta$, given in the field $\Xi$, in which elementary subregions become "standard". On surface $(\xi, \eta)$ a grid is under construction $\tilde{\omega}=\left\{\left(\xi_{i, j}, \eta_{i, j}\right), i=\overline{1, N_{1}}, j=\overline{1, N_{2}}\right\}$, which is defined by functions $x=x(\xi, \eta), y=y(\xi, \eta)$, displaying a rectangle $1 \leq \xi \leq N_{1}, 1 \leq \eta \leq N_{2}$ in the plane $(\xi, \eta)$ on a curvilinear quadrangle in the plane $(x, y)$.

For transformations

$$
\begin{aligned}
& (\xi, \eta) \rightarrow(x, y): x=x(\xi, \eta), y=y(\xi, \eta), \\
& (x, y) \rightarrow(\xi, \eta): \xi=\xi(x, y), \eta=\eta(x, y),
\end{aligned}
$$

we require the difference from zero jacobians

$$
\begin{aligned}
& J(\xi, \eta)=\frac{\partial x}{\partial \xi} \frac{\partial y}{\partial \eta}-\frac{\partial x}{\partial \eta} \frac{\partial y}{\partial \xi} \\
& \tilde{J}(x, y)=\frac{\partial \xi}{\partial x} \frac{\partial \eta}{\partial y}-\frac{\partial \xi}{\partial y} \frac{\partial \eta}{\partial x} .
\end{aligned}
$$

For convenience of perception, it is advisable to represent the local consideration of grids by considering the corresponding cells $\omega, \tilde{\omega}$. Cell nodes from $\omega^{\omega}$ we set the coordinates $\left(x_{i, j}, y_{i, j}\right),\left(x_{i, j+1}, y_{i, j+1}\right),\left(x_{i+1, j+1}, y_{i+1, j+1}\right)$, $\left(x_{i+1, j}, y_{i+1, j}\right)$.

Bypassing nodes with coordinates $\left(x_{i, j}, y_{i, j}\right)$, $\left(x_{i, j+1}, y_{i, j+1}\right),\left(x_{i+1, j+1}, y_{i+1, j+1}\right),\left(x_{i+1, j}, y_{i+1, j}\right) \quad$ should be performed counter-clockwise (when viewed along the direction of acceleration of free fall).

Continuous transformation $(x, y) \rightarrow(\xi, \eta)$ is defined on each concrete cell by the formulas

$$
\begin{gathered}
x=x(\xi, \eta)=x_{i, j}+\left(x_{i+1, j}-x_{i, j}\right) \xi+\left(x_{i, j+1}-x_{i, j}\right) \eta+ \\
+\left(x_{i+1, j}-x_{i+1, j+1}-x_{i+1, j}+x_{i, j}\right) \xi \eta, \\
y=y(\xi, \eta)=y_{i, j}+\left(y_{i+1, j}-y_{i, j}\right) \xi+\left(y_{i, j+1}-y_{i, j}\right) \eta+ \\
+\left(y_{i+1, j}-y_{i+1, j+1}-y_{i+1, j}+y_{i, j}\right) \xi \eta .
\end{gathered}
$$

The cells are convex quadrangles, where each of the angles is less than $\pi$, which ensures the positivity of the jacobian

$$
\begin{gathered}
J(\xi, \eta)=\left(x_{i+1, j}-x_{i, j}+\left(x_{i+1, j}-x_{i+1, j+1}-x_{i+1, j}+x_{i, j}\right) \eta\right) . \\
\cdot\left(y_{i+1, j+1}-y_{i, j}+\left(y_{i+1, j}-y_{i+1, j+1}-y_{i+1, j}+y_{i, j}\right) \xi\right)- \\
-\left(x_{i+1, j+1}-x_{i, j}+\left(x_{i+1, j}-x_{i+1, j+1}-x_{i+1, j}+x_{i, j}\right) \xi\right) . \\
\cdot\left(y_{i+1, j}-y_{i, j}+\left(y_{i+1, j}-y_{i+1, j+1}-y_{i+1, j}+y_{i, j}\right) \eta\right)
\end{gathered}
$$

and makes restrictions when choosing the shape of the cell.

By numbering the vertices of the cell from 1 to 4 clockwise from the node $i j$, we denote the doubled areas of the triangles $J_{1, i, j}, J_{2, i, j+1}, J_{3, i+1, j+1}, J_{4, i+1, j}$. For the node $(i, j)$ we have $J_{i, j}=\frac{1}{4}\left(J_{1}+J_{2}+J_{3}+J_{4}\right)_{i, j}$.

It is obvious that the condition for the jacobian to be positive $J$, is equivalent to the positivity of the quantities $J_{1}, J_{2}, J_{3}, J_{4}$. At least one of these quantities will be zero on the boundary of the convex mesh constructed. 
The procedure for constructing grids is connected with the consideration of the Dirichlet functional

$$
I=\iint g d x d y=\iint \frac{g}{J} d \xi d \eta
$$

$g=g_{11}+g_{22}, g_{11}=\left(\frac{\partial \xi}{\partial x}\right)^{2}+\left(\frac{\partial \xi}{\partial y}\right)^{2}$ and $g_{22}=\left(\frac{\partial \eta}{\partial x}\right)^{2}+\left(\frac{\partial \eta}{\partial y}\right)_{-}$ components of the metric tensor of the coordinate transformation.

The Euler equations for the functional (22) can be written as a system of nonlinear equations with respect to the unknown functions $x=x(\xi, \eta), y=y(\xi, \eta)$. A discrete analogue of a functional is the one obtained by summing over all four angles (triangles formed when a quadrilateral cell is partitioned by its diagonals), all grid cells, i.e.

$$
\begin{gathered}
I^{h}=\frac{1}{4} \sum_{i=1}^{N_{1}-1 N_{2}-1} \sum_{j=1}^{2}\left\{\left[\left(x_{i, j+1}-x_{i, j}\right)^{2}+\left(y_{i, j+1}-y_{i, j}\right)^{2}+\right.\right. \\
\left.+\left(x_{i, j}-x_{i+1, j}\right)^{2}+\left(y_{i, j}-y_{i+1, j}\right)^{2}\right] J_{1, i, j}^{-1}+\left[\left(x_{i+1, j+1}-x_{i, j+1}\right)^{2}+\right. \\
\left.+\left(y_{i+1, j+1}-y_{i, j+1}\right)^{2}+\left(x_{i, j+1}-x_{i, j}\right)^{2}+\left(y_{i, j+1}-y_{i, j}\right)^{2}\right] J_{2, i, j+1}^{-1}+ \\
+\left[\left(x_{i+1, j}-x_{i+1, j+1}\right)^{2}+\left(y_{i+1, j}-y_{i+1, j+1}\right)^{2}+\left(x_{i+1, j+1}-x_{i, j+1}\right)^{2}+\right. \\
\left.+\left(y_{i+1, j+1}-y_{i, j+1}\right)^{2}\right] J_{3, i+1, j+1}^{-1}+\left[\left(x_{i, j}-x_{i+1, j}\right)^{2}+\left(y_{i, j}-y_{i+1, j}\right)^{2}+\right. \\
\left.\left.+\left(x_{i+1, j}-x_{i+1, j+1}\right)^{2}+\left(y_{i+1, j}-y_{i+1, j+1}\right)^{2}\right] J_{4, i+1, j}^{-1}\right\} .
\end{gathered}
$$

Expression (23) can be obtained by replacing $x(\xi, \eta), y(\xi, \eta) \quad$ in $\quad(22) \quad$ by $\quad x^{h}(\xi, \eta), y^{h}(\xi, \eta)$ approximations by quadrature formulas of integrals over square cells in the plane $\xi, \eta$.

Solution of the system

$$
\frac{\partial I^{h}}{\partial x_{i, j}}=0, \frac{\partial I^{h}}{\partial y_{i, j}}=0
$$

gives at least one solution - a convex grid. To find it, we minimize the function $I^{h}$.

When a grid is built on $m$ - stepiteration, then $(m+1)$ - step it is determined from the solution of two linear equations written for each internal node [17]:

$$
\left\{\begin{array}{c}
\mu \frac{\partial J^{h(m)}}{\partial x_{i, j}}+\frac{\partial\left(\frac{\partial J^{h(m)}}{\partial x_{i, j}}\right)}{\partial x_{i, j}}\left(x_{i, j}^{(m+1)}-x_{i, j}^{(m)}\right)-\frac{\partial\left(\frac{\partial J^{h(m)}}{\partial x_{i, j}}\right)}{\partial y_{i, j}}\left(y_{i, j}^{(m+1)}-y_{i, j}^{(m)}\right)=0, \\
\mu \frac{\partial J^{h(m)}}{\partial x_{i, j}}+\frac{\partial\left(\frac{\partial J^{h}}{\partial y_{i, j}}\right)}{\partial x_{i, j}}\left(x_{i, j}^{(m+1)}-x_{i, j}^{(m)}\right)-\frac{\partial\left(\frac{\partial J^{h}}{\partial y_{i, j}}\right)}{\partial y_{i, j}}\left(y_{i, j}^{(m+1)}-y_{i, j}^{(m)}\right)=0,
\end{array}\right.
$$

where $\mu$ - iteration parameter, $\mu=1$.
We note that the necessary condition for a minimum of the functional is a consequence of the conformality of the transformation under consideration.

Minimization $I^{h}$ on the set of convex meshes is realized by means of a quasi-Newtonian procedure in the computational domain $(\xi, \eta)$. To do this, it is expedient to use the well-proven gradient methods, tested and described in [9] with respect to the water area of the Azov Sea and Taganrog Bay.

Change of the sign in the denominators of the integrands of the jacobian (22) - (23) functionals $J$, leads to discontinuities of the second kind, which is critical for second-order methods (quasi-newtonian procedure). To exit this situation near the degeneracy boundary $(J=0)$ coordinate transformation is performed $J$ by

$$
J^{\prime}=\max (J, \varepsilon)=(J-\varepsilon)_{+},
$$

$\varepsilon$ - a sufficiently small number, $\varepsilon>0$.

It is important to note that when generating a grid, using the above procedure, the initial approximation in the form of a convex grid is specified. If this is not the case, then the initial grid-convex grid is determined by successively solving optimization problems.

Note that since cells with numbers containing $i=1, N_{1}, j=1, N_{2}$ are boundary, then for them the position of the nodes is not optimized. Therefore, further consideration of the problem is carried out at the nodes $i=\overline{2, N_{1}-1}, j=\overline{2, N_{2}-1}$.

We introduce the notation

$$
\begin{aligned}
f\left(J_{k}\right)=\frac{1+\left(1-\frac{J_{k}}{J_{k}^{\prime}}\right)+\left(1-\frac{J_{k}}{J_{k}^{\prime}}\right)^{2}}{J_{k}^{\prime}} & =\left\{\begin{array}{c}
\frac{1}{J_{k}}, J_{k} \geq \varepsilon, \\
1+\left(1-\frac{J_{k}}{\varepsilon}\right)+\left(1-\frac{J_{k}}{\varepsilon}\right)^{2} \\
\varepsilon
\end{array}, J_{k} \leq \varepsilon,\right.
\end{aligned}
$$

Taking into account the last equality, expression (23) can be represented in the following form

$$
I^{h^{*}}=\sum_{i=2}^{N_{1}-1 N_{2}-1}\left[g_{j=2} f\left(J_{1}\right)+g_{2} f\left(J_{2}\right)+g_{3} f\left(J_{3}\right)+g_{4} f\left(J_{4}\right)\right]_{i, j},
$$

where

$$
\begin{aligned}
& J_{1, i, j}=\left(x_{i+1, j}-x_{i, j}\right)\left(y_{i, j+1}-y_{i, j}\right)-\left(y_{i+1, j}-y_{i, j}\right)\left(x_{i, j+1}-x_{i, j}\right), \\
& J_{2, i, j}=\left(x_{i+1, j}-x_{i, j}\right)\left(y_{i, j}-y_{i, j-1}\right)-\left(y_{i+1, j}-y_{i, j}\right)\left(x_{i, j}-x_{i, j-1}\right), \\
& J_{3, i, j}=\left(x_{i, j}-x_{i-1, j}\right)\left(y_{i, j}-y_{i, j-1}\right)-\left(y_{i, j}-y_{i-1, j}\right)\left(x_{i, j}-x_{i, j-1}\right), \\
& J_{4, i, j}=\left(x_{i, j}-x_{i-1, j}\right)\left(y_{i, j+1}-y_{i, j}\right)-\left(y_{i, j}-y_{i-1, j}\right)\left(x_{i, j+1}-x_{i, j}\right), \\
& g_{1, i, j}=\frac{\left(x_{i, j+1}-x_{i, j}\right)^{2}+\left(y_{i, j+1}-y_{i, j}\right)^{2}+\left(x_{i, j}-x_{i+1, j}\right)^{2}+\left(y_{i, j}-y_{i+1, j}\right)^{2}}{4}, \\
& g_{2, i, j}=\frac{\left(x_{i+1, j}-x_{i, j}\right)^{2}+\left(y_{i+1, j}-y_{i, j}\right)^{2}+\left(x_{i, j}-x_{i, j-1}\right)^{2}+\left(y_{i, j}-y_{i, j-1}\right)^{2}}{4}, \\
& g_{3, i, j}=\frac{\left(x_{i, j-1}-x_{i, j}\right)^{2}+\left(y_{i, j-1}-y_{i, j}\right)^{2}+\left(x_{i, j}-x_{i-1, j}\right)^{2}+\left(y_{i, j}-y_{i-1, j}\right)^{2}}{4}, \\
& g_{4, i, j}=\frac{\left(x_{i-1, j}-x_{i, j}\right)^{2}+\left(y_{i-1, j}-y_{i, j}\right)^{2}+\left(x_{i, j}-x_{i, j+1}\right)^{2}+\left(y_{i, j}-y_{i, j+1}\right)^{2}}{4} .
\end{aligned}
$$


Solution of a system of algebraic equations of the form (24) for $I^{h^{*}}$ representing the necessary condition for the minimum of the functional $I^{h^{*}}$, gives at least one solution (convex grid).

Further, inordertominimize $I^{h^{*}}$ they used the same quasi-Newtonian procedure as when minimizing the function $I^{h}$.

To determine the gradient components of a discrete functional $I^{h^{*}}$ represented locally in the point $\left(x_{i, j}, y_{i, j}\right)$, the following derivatives were required:

$$
\begin{aligned}
& \left(\frac{\partial J_{1}}{\partial x}\right)_{i, j}=-\left(y_{i, j+1}-y_{i, j}\right)+\left(y_{i+1, j}-y_{i, j}\right)=y_{i+1, j}-y_{i, j+1}, \\
& \left(\frac{\partial J_{2}}{\partial x}\right)_{i, j}=-\left(y_{i, j}-y_{i, j-1}\right)-\left(y_{i+1, j}-y_{i, j}\right)=y_{i, j}-y_{i+1, j} \text {, } \\
& \left(\frac{\partial J_{3}}{\partial x}\right)_{i, j}=\left(y_{i, j}-y_{i, j}\right)-\left(y_{i, j}-y_{i-1, j}\right)=y_{i-1, j}-y_{i, j-1}, \\
& \left(\frac{\partial J_{4}}{\partial x}\right)_{i, j}=\left(y_{i, j+1}-y_{i, j}\right)+\left(y_{i, j}-y_{i-1, j}\right)=y_{i, j+1}-y_{i-1, j} \text {, } \\
& \left(\frac{\partial g_{1}}{\partial x}\right)_{i, j}=\frac{1}{2}\left\{\left(-x_{i, j+1}+x_{i, j}\right)+\left(x_{i, j}-x_{i+1, j}\right)\right\}, \\
& \left(\frac{\partial g_{2}}{\partial x}\right)_{i, j}=\frac{1}{2}\left\{\left(-x_{i+1, j}+x_{i, j}\right)+\left(x_{i, j}-x_{i, j-1}\right)\right\} \text {, } \\
& \left(\frac{\partial g_{3}}{\partial x}\right)_{i, j}=\frac{1}{2}\left\{\left(-x_{i, j-1}+x_{i, j}\right)+\left(x_{i, j}-x_{i-1, j}\right)\right\} \text {, } \\
& \left(\frac{\partial g_{4}}{\partial x}\right)_{i, j}=\frac{1}{2}\left\{\left(-x_{i-1, j}+x_{i, j}\right)+\left(x_{i, j}-x_{i, j+1}\right)\right\}, \\
& \left(\frac{\partial J_{1}}{\partial y}\right)_{i, j}=-\left(x_{i+1, j}-x_{i, j}\right)+\left(x_{i, j+1}-x_{i, j}\right)=x_{i, j+1}-x_{i+1, j} \text {, } \\
& \left(\frac{\partial J_{2}}{\partial y}\right)_{i, j}=\left(x_{i+1, j}-x_{i, j}\right)+\left(x_{i, j}-x_{i, j-1}\right)=x_{i+1, j}-x_{i, j-1} \text {, } \\
& \left(\frac{\partial J_{3}}{\partial y}\right)_{i, j}=\left(x_{i+1, j}-x_{i, j}\right)-\left(x_{i+1, j}-x_{i+1, j-1}\right)=x_{i+1, j-1}-x_{i, j} \text {, } \\
& \left(\frac{\partial J_{4}}{\partial y}\right)_{i, j}=-\left(x_{i, j}-x_{i-1, j}\right)-\left(x_{i, j+1}-x_{i, j}\right)=x_{i-1, j}-x_{i, j} \text {, } \\
& \left(\frac{\partial g_{1}}{\partial y}\right)_{i, j}=\frac{1}{2}\left\{\left(-y_{i, j+1}+y_{i, j}\right)+\left(y_{i, j}-y_{i+1, j}\right)\right\}, \\
& \left(\frac{\partial g_{2}}{\partial y}\right)_{i, j}=\frac{1}{2}\left\{\left(-y_{i+1, j}+y_{i, j}\right)+\left(y_{i, j}-y_{i, j-1}\right)\right\}, \\
& \left(\frac{\partial g_{3}}{\partial y}\right)_{i, j}=\frac{1}{2}\left\{\left(-y_{i, j-1}+y_{i, j}\right)+\left(y_{i, j}-y_{i-1, j}\right)\right\} \text {, } \\
& \left(\frac{\partial g_{4}}{\partial y}\right)_{i, j}=\frac{1}{2}\left\{\left(-y_{i-1, j}+y_{i, j}\right)+\left(y_{i, j}-y_{i, j+1}\right)\right\} \text {, } \\
& \left(f_{J}^{\prime}\right)_{k}=\frac{1+2\left(1-\frac{J_{k}}{J_{k}^{\prime}}\right)}{\left(J_{k}^{\prime}\right)^{2}}=\left\{\begin{array}{c}
-\frac{1}{\left(J_{k}\right)^{2}}, J_{k} \geq \varepsilon, \\
1+2\left(1-\frac{J_{k}}{\varepsilon}\right) \\
-\frac{\varepsilon^{2}}{\varepsilon^{2}}, J_{k} \leq \varepsilon, k=\overline{1,4} .
\end{array}\right.
\end{aligned}
$$

Taking into account the expressions obtained, we find the components of the gradient of the functional

$$
I^{h^{*}}=\left\{g_{1} f\left(J_{1}\right)+g_{2} f\left(J_{2}\right)+g_{3} f\left(J_{3}\right)+g_{4} f\left(J_{4}\right)\right\}_{i j},
$$

соответствующие декартовым координатам $\left(x_{i, j}, y_{i, j}\right)$ node $(i, j)$ :

$$
\begin{aligned}
& \left(\frac{\partial I^{h^{*}}}{\partial x}\right)_{i, j}=\left(\sum_{k=1}^{4} \frac{\partial g_{k}}{\partial x} f\left(J_{k}\right)+g_{k} \frac{\partial f\left(J_{k}\right)}{\partial J_{k}} \frac{\partial J_{k}}{\partial x}\right)_{i, j}, \\
& \left(\frac{\partial I^{h^{*}}}{\partial y}\right)_{i, j}=\left(\sum_{k=1}^{4} \frac{\partial g_{k}}{\partial y} f\left(J_{k}\right)+g_{k} \frac{\partial f\left(J_{k}\right)}{\partial J_{k}} \frac{\partial J_{k}}{\partial y}\right)_{i, j} .
\end{aligned}
$$

Expressions (27) - (28) are determined by substituting in them the corresponding values of the derivatives from.

In Fig. 1 schematically represents the calculated grid (Azov Sea $-200 \times 80$, Taganrog Bay $-108 \times 40$ knots), the number of cells of which significantly exceeds the analogue of its predecessors.

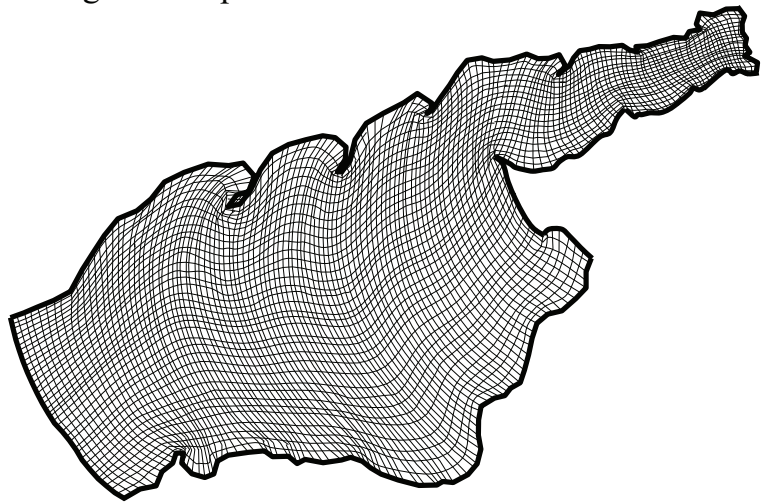

Fig. 1. Building a grid of physical area.

\subsection{Approximation of the sediment transport equation using the finite element method}

Equation (15) can be written in the form

$$
\begin{gathered}
-\left(\frac{\partial}{\partial x}\left(k^{n-1} \frac{\tau_{b c}}{\sin \varphi_{0}} \frac{\partial H^{n}}{\partial x}\right)-\frac{\partial}{\partial y}\left(k^{n-1} \frac{\tau_{b c}}{\sin \varphi_{0}} \frac{\partial H^{n}}{\partial y}\right)\right)+ \\
+\frac{(1-\varepsilon)}{\tau} H^{n}=\frac{(1-\varepsilon)}{\tau} H^{n-1}-\left(\frac{\partial}{\partial x}\left(k^{n-1} \tau_{b x}\right)+\frac{\partial}{\partial y}\left(k^{n-1} \tau_{b y}\right)\right)
\end{gathered}
$$

and note that in the new coordinates

$$
\begin{gathered}
H^{n} \equiv H^{n}(x(\xi, \eta), y(\xi, \eta)), H^{n-1} \equiv H^{n}(x(\xi, \eta), y(\xi, \eta)), \\
k^{n-1} \equiv k^{n-1}(x(\xi, \eta), y(\xi, \eta)) .
\end{gathered}
$$

Using the expressions for the derivatives

$$
\frac{\partial H}{\partial x}=\frac{\partial H}{\partial \xi} \frac{\partial \xi}{\partial x}+\frac{\partial H}{\partial \eta} \frac{\partial \eta}{\partial x}, \frac{\partial H}{\partial y}=\frac{\partial H}{\partial \xi} \frac{\partial \xi}{\partial y}+\frac{\partial H}{\partial \eta} \frac{\partial \eta}{\partial y}
$$

for (29) we find 


$$
\begin{gathered}
-\frac{\partial}{\partial \xi}\left\{k^{n-1} \frac{\tau_{b c}}{\sin \varphi_{0}}\left(g_{11} \frac{\partial H^{n}}{\partial \xi}+g_{12} \frac{\partial H^{n}}{\partial \eta}\right)\right\}- \\
-\frac{\partial}{\partial \eta}\left\{k^{n-1} \frac{\tau_{b c}}{\sin \varphi_{0}}\left(g_{22} \frac{\partial H^{n}}{\partial \eta}+g_{12} \frac{\partial H^{n}}{\partial \xi}\right)\right\}+\frac{(1-\varepsilon)}{\tau} H^{n}= \\
=\frac{(1-\varepsilon)}{\tau} H^{n-1}-\left(\frac{\partial}{\partial \xi}\left(\left(k^{n-1} \tau_{b x}\right) \frac{\partial \xi}{\partial x}+\left(k^{n-1} \tau_{b y}\right) \frac{\partial \xi}{\partial y}\right)+\right. \\
\left.+\frac{\partial}{\partial \eta}\left(\left(k^{n-1} \tau_{b x}\right) \frac{\partial \eta}{\partial x}+\left(k^{n-1} \tau_{b y}\right) \frac{\partial \eta}{\partial y}\right)\right) .
\end{gathered}
$$

We define the scalar product

$$
\begin{gathered}
(f, g)=\iint_{D} f(x, y) g(x, y) d x d y= \\
=\iint_{\Xi} f(x(\xi, \eta), y(\xi, \eta)) g(x(\xi, \eta), y(\xi, \eta)) J d \xi d \eta
\end{gathered}
$$

We multiply the equation (30) by the rule (31) by an arbitrary function $v(x(\xi, \eta), y(\xi, \eta))$. We get

$$
\begin{gathered}
-g_{11} \iint_{\Xi} \frac{\partial}{\partial \xi}\left(k^{n-1} \frac{\tau_{b c}}{\sin \varphi_{0}} \frac{\partial H^{n}}{\partial \xi}\right) v J d \xi d \eta- \\
-g_{22} \iint_{\Xi} \frac{\partial}{\partial \eta}\left(k^{n-1} \frac{\tau_{b c}}{\sin \varphi_{0}} \frac{\partial H^{n}}{\partial \eta}\right) v J d \xi d \eta- \\
-g_{12} \iint_{\Xi}\left(\frac{\partial}{\partial \xi}\left(k^{n-1} \frac{\tau_{b c}}{\sin \varphi_{0}} \frac{\partial H^{n}}{\partial \eta}\right)+\frac{\partial}{\partial \eta}\left(k^{n-1} \frac{\tau_{b c}}{\sin \varphi_{0}} \frac{\partial H^{n}}{\partial \xi}\right)\right) v J d \xi d \eta+ \\
+\frac{(1-\varepsilon)}{\tau} \iint_{\Xi} H^{n} v J d \xi d \eta=\frac{(1-\varepsilon)}{\tau} \iint_{\Xi} H^{n-1} v J d \xi d \eta- \\
-\frac{\partial \xi}{\partial x} \iint_{\Xi}\left(\frac{\partial}{\partial \xi}\left(k^{n-1} \tau_{b x}\right)\right) v J d \xi d \eta-\frac{\partial \xi}{\partial y} \iint_{\Xi} \frac{\partial}{\partial \xi}\left(k^{n-1} \tau_{b y}\right) v J d \xi d \eta- \\
\frac{\partial \eta}{\partial x} \iint_{\Xi}\left(\frac{\partial}{\partial \eta}\left(k^{n-1} \tau_{b x}\right)\right) v J d \xi d \eta-\frac{\partial \eta}{\partial y} \iint_{\Xi} \frac{\partial}{\partial \eta}\left(k^{n-1} \tau_{b y}\right) v J d \xi d \eta .
\end{gathered}
$$

Consider the approximation of functions defined on a polygonal domain, $D$ using piecewise linear basis functions. Area $D$ undergo a triangulation.

The set of basis functions $\varphi_{i j}$ is defined in the standard way.

The required approximate solution $\tilde{H}^{n}$ of the problem under consideration can be represented by expanding in terms of the basis functions

$$
\tilde{H}^{n}\left(x(\xi, \eta), y(\xi, \eta), t_{n-1}\right)=\sum_{i=1}^{N_{1}-1 N_{j}-1} \sum_{j=1} z_{i j} \varphi_{i j}(x(\xi, \eta), y(\xi, \eta)) .
$$

To describe the computational procedures for finding finitely-elementary solutions of the initial-boundary problem of sediment transport with allowance for (32), it is convenient to write down its matrix analogue:

$$
\begin{gathered}
-A z+\frac{(1-\varepsilon)}{\tau} B z=C, \\
z=\left(z_{i j}\right),
\end{gathered}
$$

$$
\begin{gathered}
A=\left(A_{i j, k l}\right), A_{i j, k l}=g_{11} \iint_{\Xi} \frac{\partial}{\partial \xi}\left(k^{n-1} \frac{\tau_{b c}}{\sin \varphi_{0}} \frac{\partial \varphi_{k l}}{\partial \xi}\right) \varphi_{i j} J d \xi d \eta+ \\
+g_{22} \iint_{\Xi} \frac{\partial}{\partial \eta}\left(k^{n-1} \frac{\tau_{b c}}{\sin \varphi_{0}} \frac{\partial \varphi_{k l}}{\partial \eta}\right) \varphi_{i j} J d \xi d \eta+ \\
+g_{12} \iint_{\Xi}\left(\frac{\partial}{\partial \xi}\left(k^{n-1} \frac{\tau_{b c}}{\sin \varphi_{0}} \frac{\partial \varphi_{k l}}{\partial \eta}\right)+\frac{\partial}{\partial \eta}\left(k^{n-1} \frac{\tau_{b c}}{\sin \varphi_{0}} \frac{\partial \varphi_{k l}}{\partial \xi}\right)\right) \varphi_{i j} J d \xi d \eta \\
B=\left(B_{i j, k l}\right), B_{i j, k l}=\frac{(1-\varepsilon)}{\tau} \iint_{\Xi} \varphi_{k l} \varphi_{i j} J d \xi d \eta, \\
-\frac{\partial \xi}{\partial x} \iint_{\Xi}\left(\frac{\partial}{\partial \xi}\left(k^{n-1} \tau_{b x}\right)\right) \varphi_{i j} J d \xi d \eta-\frac{\partial \xi}{\partial y} \iint_{\Xi} \frac{\partial}{\partial \xi}\left(k^{n-1} \tau_{b y}\right) \varphi_{i j} J d \xi d \eta- \\
-\frac{\partial \eta}{\partial x} \iint_{\Xi}\left(\frac{\partial}{\partial \eta}\left(k^{n-1} \tau_{b x}\right)\right) \varphi_{i j} J d \xi d \eta-\frac{\partial \eta}{\partial y} \iint_{\Xi} \frac{\partial}{\partial \eta}\left(k^{n-1} \tau_{b y}\right) \varphi_{i j} J d \xi d \eta, \\
i, k=\overline{1, N_{1}}, j, l=1, N_{2} .
\end{gathered}
$$

The grid equation (33) is actually the SLAE for the unknown vector

$$
\left(-A+\frac{(1-\varepsilon)}{\tau} \cdot B\right) \cdot z=C
$$

with a global stiffness matrix of the form $\left(-A+\frac{(1-\varepsilon)}{\tau} B\right)$ [19-20].

We note that after carrying out a sequential computational procedure for the compilation and solution of equations (34), we obtain a solution of the initial-boundary value problem on all time layers of interest to us $n=1, \ldots, N$.

\section{Conclusions}

On the optimal boundary-adaptive grid constructed on the basis of minimization of the generalized Dirichlet functional, a discrete two-dimensional mathematical model for a linearized model of sediment transport in coastal systems that satisfies the basic conservation laws is constructed by the finite element method. A finite element approximation has been implemented in relation to the water area of the Azov Sea and the Taganrog Bay and, thus, a system of finite-element equations has been prepared for the subsequent numerical modeling of sediment transport processes.

This work was supported by the RNF (project code 17-1101286).

\section{References}

1. A.I. Sukhinov, V.V. Sidoryakina, The theory of operators, complex analysis and mathematical modeling. Abstracts of the XIII International Scientific Conference, 184-185 (2016) 
2. A.I. Sukhinov, V.V. Sidoryakina, Bulletin of the Taganrog State Pedagogical Institute, № 2, 270-274 (2016)

3. A.I. Sukhinov, V.V. Sidoryakina, Bulletin of the Taganrog State Pedagogical Institute, № 2, 245-249, (2017)

4. A.I. Sukhinov, V.V. Sidoryakina, A.A. Sukhinov, Bulletin of the Don State Technical University, 17, № 1 (88), 5-17 (2017)

5. V.V. Sidoryakina, A.I. Sukhinov, Computational Mathematics and Mathematical Physics, 57, № 6, 978-994 (2017)

6. V.V. Sidoryakina, A.I. Sukhinov, Zhurnal Vychislitel'noi Matematiki i Matematicheskoi Fiziki, 57, № 6, 985-1002 (2017)

7. A.I. Sukhinov, A.E. Chistyakov, E.V. Alekseenko, Mathematical Models and Computer Simulations, 23, №. 3, 3-21 (2011)

8. A.I. Sukhinov, A.A. Sukhinov, Parallel Computational Fluid Dynamics, Mutidisciplinary Applications, Prcoceedings of Parallel CFD 2004 Conference, 231-238 (2005)

9. V.S. Vasiliev, A.I. Sukhinov, Matem. Modelling, 15, № 10, 17-34 (2003)

10. I.O. Leontyev, Coastal dynamics: waves, moving streams, deposits drifts (GEOS, Moscow, 2001)

11. A.I. Sukhinov, A.E. Chistyakov, E.A. Protsenko, News of SFedU. Technical science, № 8 (121), 32-44 (2011)

12. A.I. Sukhinov, A.E. Chistyakov, E.A. Protsenko, Matematicheskoe Modelirovanie, 25, № 12, 65-82, (2013)

13. E. Alekseenko, B. Roux, A. Sukhinov, R. Kotarba, D. Fougere, Nonlinear Processes in Geophysics, 20, № 2, 189-198 (2013)

14. A.I. Sukhinov, A.E. Chistyakov, E.A. Protsenko, Math. Models Comput. Simul., 6, № 4, 351-363 (2014)

15. A.I. Sukhinov, A.E.Chistyakov, E.A. Protsenko, Numerical Methods and Programming, 15, № 4, 610-620 (2014)

16. S.A.Ivanenko, Zhurnal Vychislitel'noi Matematiki i Matematicheskoi Fiziki, 28, № 10, 1498-1506 (1988)

17. G.I. Marchuk, V.I. Agoshkov, Introduction to projection-grid methods (Science. The main edition of physics and mathematics, Moscow, 1981)

18. I.A. Vaseva, A.V. Kofanov, V.D. Liseikin, Yu.V. Likhanova, A.M. Kharitonchik, Vychislitel'noi Matematiki i Matematicheskoi Fiziki, 50, № 1, 99117 (2010)

19. A.A. Samarskii, A.V. Gulin, Numerical methods of mathematical physics. 2-nd ed. (The scientific world, Moscow, 2003)

20. O.M. Belotserkovsky, Numerical modeling at mechanics continuous environments (Fizmatlit, Moscow, 2004) 